Nouvelles perspectives en sciences sociales

Revue internationale de systémique complexe et d'études relationnelles

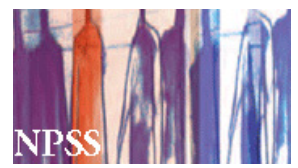

\title{
L'économiste dans la Cité: ingénierie et expertise
}

\section{Michel Armatte}

Volume 6, numéro 2, juin 2011

Sur le thème : « Les économistes dans la Cité »

URI : https://id.erudit.org/iderudit/1005772ar

DOI : https://doi.org/10.7202/1005772ar

Aller au sommaire du numéro

Éditeur(s)

Prise de parole

ISSN

1712-8307 (imprimé)

1918-7475 (numérique)

Découvrir la revue

Citer cet article

Armatte, M. (2011). L'économiste dans la Cité: ingénierie et expertise. Nouvelles perspectives en sciences sociales, 6(2), 97-131. https://doi.org/10.7202/1005772ar

\section{Résumé de l'article}

À la vision mythique et autiste d'une science économique pure réduite à sa version académique, et déconnectée des réalités, nous opposons une autre vision de l'économie comme ingénierie, agissant sur cette réalité et la transformant à son image. Pour en rendre compte nous développons des exemples empruntés à la finance, à l'économétrie, ou à la prospective climatique, et nous mobilisons des notions propres à l'histoire et la sociologie des sciences, et plus précisément à la tradition des sciences studies comme celles de performativité (Callon) et de régimes de savoirs et d'expertise (Pestre), ce qui nous permet de décrire différentes formes d'intervention de l'économiste dans la cité. 


\title{
L'économiste dans la Cité: ingénierie et expertise
}

\author{
Michel Armatte \\ Université Paris Dauphine \& Centre A. Koyré
}

$\mathbf{U}$

n débat autour du thème " des économistes dans la Cité " tel qu'il fut organisé par le LEREPS de Toulouse dans la Journée du 8 janvier 2010 doit commencer, nous semble-t-il, par dresser un rapide portrait des faux semblants de la discipline académique, c'est-à-dire de la vision réflexive dominante que la discipline économique a d'elle-même dans les enceintes académiques qui sont celles de l'université et des organismes publics de recherche. Par contraste avec ce mythe - et ce contraste a quelques fois été traité d'autiste (cf. infra), on peut imaginer ce que serait une autre vision dans laquelle le rôle d'acteur de l'économiste dans la cité peut être pris en compte. Nous proposons ci-dessous une vision alternative, dans laquelle on considère la science économique comme une ingénierie, agissant sur la société réelle avec ses armes théoriques et techniques, via des dispositifs socio-cognitifs, et à des fins d'expertise, de gestion et de contrôle. Il est alors intéressant de s'inspirer des sciences studies pour dégager, comme dans les autres sciences, des régimes de savoirs articulant sciences et société, et formes dominantes successives de l'expertise. Des exemples empruntés à la finance, à l'économétrie, ou à la prospective climatique nous donneront une idée de ce 
que l'économie partage avec les autres sciences dans ce rapport d'expertise, et de ce qui reste spécifique de la démarche économique. Il en ressortira une autre vision réflexive sur l'économie qui devrait aussi entraîner une autre vision éthique et politique.

\section{Illusions d'une science académique}

L'économie comme science (economics) est pour l'essentiel une création de la seconde moitié du XX $\mathrm{XX}^{\mathrm{e}}$ siècle, ayant succédé à l'économie politique qui s'affirma tout au long du siècle précédent comme une science morale autant positive que prescriptive, puis à l'économique rationnelle qui se vivait comme une science analogue à la mécanique rationnelle, pure et appliquée. Autant dire que son statut de science, en référence bien souvent aux sciences de la nature, a toujours été problématique. Comme l'est aussi le rapport à son objet, l'économie "réelle " (economy) définie d'abord comme l'ensemble des phénomènes concernant la production, la distribution et la consommation des richesses, avant que ne triomphe une conception à la fois plus étroite dans sa définition mais plus large dans son ambition, en tout cas plus formelle car issue des hypothèses néoclassiques qui forment le main stream de la discipline. Aujourd'hui la science économique dominante ne traite plus des phénomènes les plus divers observés dans le fonctionnement marchand des sociétés, mais des conséquences que l'on peut déduire d'un jeu d'hypothèses et de principes d'optimisation caractérisant les choix d'individus supposés rationnels, et qui re-constituent une économie fictive mais référentielle.

L'objet de l'économie standard est en effet un objet artificiel - une société d'homo æconomicus interchangeables - qui n'a que peu de rapport avec nos sociétés, même si on en isole ce qui relève des échanges marchands. La science économique standard travaille sur un ensemble d'objets idéalisés, voire idolâtrés, qui sont des individus, similaires et non structurés par des valeurs et des dotations différentes, rationnels dans leurs décisions toujours conformes à un principe de maximisation de leur utilité, ne se 
rencontrant que sur des marchés où ils échangent des biens en certaines quantités et prix, déterminés par le seul mécanisme d'équilibre (général ou partiel) que l'on peut déduire de l'efficience supposée de ces marchés. L'objet de l'économie standard est alors restreint aux effets des variantes de cette économie fictive que l'on peut construire sur la base de ces quelques principes individualisme méthodologique, rationalité des agents, existences de fonctions d'utilité, existence d'un marché efficient, existence d'un équilibre réalisant un optimum de Pareto - en faisant varier quelques conditions annexes sur la symétrie des informations, sur la nature des anticipations, sur la perfection du marché.

Cette économie standard est également caractérisée par une méthode qui est celle de la modélisation mathématique. Celle-ci peut se résumer par les éléments suivants :

1. Une quantification des grandeurs du phénomène économique étudié sur la base de conventions sociales et logiques, et de dispositifs de mesure relevant soit d'institutions nationales (enquêtes statistiques, comptabilité nationale) européennes ou mondiales, soit plus récemment de dispositifs expérimentaux.

2. Une formalisation de relations (équations) entre ces grandeurs établies par un modèle que l'économiste (ou un collectif) a construit :

a. sur les bases comptables de relations postulées entre grandeurs;

b. sur les bases axiomatiques des principes de rationalité;

c. sur les bases de relations de comportement supposées d'agents représentatifs.

3. La validation empirique du modèle par estimation et tests statistiques de ses paramètres sur des séries historiques, ou par calibration sur une base de comptabilité sociale.

Ces deux composantes épistémologiques brièvement évoquées - l'objet et la méthode - forment le noyau de la pensée économique savante selon les canons académiques. Toute réflexivité par rapport à ce programme est en général rejetée aux marges de son dispositif : les problèmes qui surgissent de l'inadéquation des 
économies réelles et de l'économie rêvée par le main stream constituent des externalités par rapport aux mécanismes retenus, positives ou négatives, qui seront laissées à quelques spécialistes ou aux différentes école dites hétérodoxes (institutionnalistes, conventionnalistes, école de la régulation), aux études réflexives développées par l'épistémologie économique, l'histoire des faits économiques et l'histoire de la pensée économique, voire à des disciplines en formation sur les marges (socio-économie, anthropologie économique, éco-développement...).

Assez régulièrement, surgit une crise plus ou moins profonde à l'intérieur même des institutions académiques, dans laquelle ce paradigme dominant de la science économique est mis en cause. Une des crises les plus récentes eut lieu au début des années 2000 dans l'enseignement supérieur français. Des étudiants, et pas des moins qualifiés puisqu'ils provenaient des formations universitaires les plus prestigieuses et sélectives (École Normale, École des Mines, Universités Paris 1, Dauphine, Nanterre...), ont repris un questionnement couramment fait à l'économie, aussi bien par les étudiants que dans l'espace public et les média. $Y$ a-t-il vraiment un rapport entre la science économique académique, celle des chercheurs, celle que l'on enseigne à l'Université, et les questions économiques de notre temps dont le traitement sétale sur les premières pages de nos quotidiens et des journaux télévisés? Et ils ont répondu par cette formule choc : l'enseignement des sciences économiques est profondément autiste, c'est-à-dire totalement déconnecté des réalités socio-économiques. Leur mouvement, s'élargissant et suscitant nationalement un rapport au Ministre ${ }^{1}$, puis devenant international sous le nom de post autistic economics ${ }^{2}$ dénonçait un divorce inquiétant entre la science apprise et les questions économiques traitées par l'actualité, qu'ils attribuaient à deux facteurs principaux: d'abord à l'omniprésence du seul courant de pensée néoclassique et libéral comme fonds doctrinaire, ensuite à l'enfermement dans des routines d'analyses for-

$1 \quad$ Jean-Paul Fitoussi, L'enseignement supérieur des sciences économiques en question, Rapport au Ministre de l'Éducation nationale, Paris, Fayard, 2001.

2 Real-World Economics Review, http://www.paecon.net/ consulté en janvier 2011. 
malisées ou modélisées enchaînées à l'infini. Ces étudiants, soutenus par quelques enseignants, réclamaient, pour répondre à la complexité et aux défis des problèmes économiques et sociaux d'aujourd'hui, un retour aux faits économiques plutôt qu'à l'étude de fictions, avec un recul critique et une approche pluraliste des faits de société. Les mathématiques avaient un rôle important dans cette critique puisqu'elles supportaient les opérations dénoncées de coupure entre monde académique et monde réel, par le fait même de construire un monde de substitution artificiel, simplifié par l'axiomatique et débarrassé des questions de pertinence et d'efficacité politiques et sociales. En permettant l'enfermement dans un jeu purement logique de raisonnements hypothético-déductifs pouvant se poursuivre indéfiniment, et n'épuisant jamais la combinatoire des spécifications formelles d'hypothèses et de fermetures possibles du système étudié, les modélisations mathématiques fabriquaient cette situation " autiste » de la discipline. Les étudiants ne réclamaient pas que l'on s'abstienne du recours aux modèles mathématiques mais que, pour un problème donné, on achève le détour méthodologique pour en revenir à la question de départ, et que l'approche modélisée ne soit pas le seul point de vue offert sur cette question, qu'il soit au minimum discuté et relayé par d'autres types d'analyses (historiques, institutionnelles, etc.).

Nous avons soutenu ce mouvement de la critique autiste, à la fois comme enseignant et comme historien. En effet elle pointe parfaitement les critiques que l'on peut faire à la formation des économistes aujourd'hui : l'enfermement dans un paradigme dominant néoclassique plonge l'étudiant dans l'analyse de mondes artificiels qui ne répond pas à ce qu'il voit, ou devine être la complexité d'une société marchande. Il faut non pas remplacer cette approche néolibérale par une autre, mais la relativiser à l'aide de plusieurs autres approches des mêmes phénomènes. Le seul antidote à l'effet autistique autant qu'idéologique de cette imposition est dans le pluralisme des approches. A minima convient-il de confronter cette approche hypothético-déductive à des images produites par des descriptions variées et contextua- 
lisées, produites par les sciences sociales, les différents médias, et le débat politique et citoyen. Face au risque de s'enliser dans la syntaxe des modèles et dans les heuristiques qu'ils autorisent, il faut compenser ce risque d'enfermement autiste par un large appel à des connaissances d'un autre type sur l'activité économique et ses problématiques. Lecture de la presse, témoignages, lecture de rapports, analyse de controverses sont alors des méthodologies complémentaires à la modélisation indispensables si l'on veut construire un pont solide entre economics et economy.

On objectera facilement que cette approche alternative peut aider à un tel rapprochement mais que l'économie descriptive n'est plus depuis longtemps au programme des formations universitaire parce qu'elle est trop complexe et trop difficile à enseigner. Et qu'une bonne formation théorique - plus simple à enseigner de façon canonique (modélisations microéconomiques, modèle IS-LM, etc.) donne toutes les clés pour comprendre les enjeux et les décisions de toute politique économique. Et que ce qui compte pour les progrès de la discipline c'est l'enchaînement des idées, des théories. On voudrait démontrer dans ce qui suit que cette vision académique d'une science économique réduite à ses principes et résultats théoriques, qu'ils appartiennent ou non au main stream, est extrêmement réductrice et inopérante dès lors que l'on veut rendre compte non pas de ses seuls effets cognitifs sur la connaissance des mécanismes fondamentaux, isolés comme économiques, mais de ses effets sur l'innovation, l'organisation, la gestion des dispositifs économiques qui performent notre société. En bref une vision de la science comme ingénierie est la seule qui permette de prendre en compte l'économiste dans la cité.

\section{La science comme ingénierie}

La terminologie de sciences économiques n'apparaît que dans l'après guerre et les filières économiques de l'Université ne prennent ce nom en France qu'au début des années 1960. Au-delà de l'évolution terminologique, l'activité des économistes - ingé- 
nieurs ou pas - a pris la forme d'une ingénierie. Nous voulons dire par là que la mutation de l'économie politique à la science économique s'est établie non seulement $1^{\circ}$ ) sur la base d'une unification de son corps de doctrine en un paradigme dominant (néoclassique), mais qu'elle a profité aussi $2^{\circ}$ ) d'une révolution dans la méthodologie de raisonnement et d'imposition de la preuve, en particulier en passant par des canons de la déduction mathématique ou/et de la validation statistique et $3^{\circ}$ ) d'une intégration de ses inventions théoriques dans des innovations socio-économiques lui permettant de traduire des principes ou des résultats dans des dispositifs de gestion des activités économiques $4^{\circ}$ ) et enfin d'un positionnement particulier de l'expertise économique dans des think tank et des agences étatiques. Ce sont ces quatre volets qui constituent pour nous une ingénierie.

La connexion entre monde académique et monde réel se fait la plupart du temps à double sens : la plupart des savoirs académiques se construisent dans des lieux de recherche pour répondre à des problèmes qui proviennent d'une demande politique et sociale; ils ne sont pas forcément nouveaux mais se posent dans les conditions particulières ou à des échelles nouvelles dans le monde réel, ce qui justifie qu'ils surgissent dans l'agenda politique et dans l'espace public. Nous avons montré dans un récent ouvrage $^{3}$ que les conditions particulières des grandes crises des années 1890 et 1930, puis celles de la seconde guerre mondiale des années 1940, ont constitué des circonstances exceptionnelles, qui sont bien différentes de celles des marchés de concurrence pure et parfaite de nos manuels, et qui ont formé la matrice des principales innovations économiques du $\mathrm{XX}^{\mathrm{e}}$ siècle telles que la recherche opérationnelle, l'économétrie ou la théorie des jeux. À l'inverse, ces innovations, en s'intégrant dans des dispositifs techniques et sociaux, ont profondément modifié les mécanismes et les rapports de la production de biens et services au XX $\mathrm{XX}^{\mathrm{e}}$ siècle : réglementation puis déréglementation des marchés de biens et services, indexation des salaires sur le mouvement des prix ou au

3 Michel Armatte, La science économique comme ingénierie. Quantification et modélisation, Paris, Presses des Mines, 2010. 
contraire libéralisation du marché du travail, traitements du chômage et gestion de la population qui en vit les effets... L'économie réelle se voit ainsi " performée " par la science économique devenue ingénierie.

Le concept de performativité mis en avant par Michel Callon ${ }^{4}$ généralise ce que dans nos travaux ${ }^{5}$ nous avions appelé les propriétés pragmatiques des objets formels de la statistique, et qui provoque leur transport et leur enrôlement d'une problématique disciplinaire à une autre. Michel Callon fait dériver ce concept des théories linguistiques de Austin, Peirce et Morris que l'on peut rattacher au "tournant pragmatique ", et il englobe tous les cas dans lesquels les énoncés, modèles et théories scientifiques n'ont pas principalement une portée descriptive mais sont activement engagés dans la (re)construction de la réalité qu'ils décrivent. Cette définition un peu attrape-tout de la performativité permet d'y inclure les deux cas limites de la prescription caractérisé par un acte volontaire et routinisé dans lequel les énoncés qui disent ce qu'il faut faire sont suivis d'une exécution, et de la prophétie autoréalisatrice pour laquelle la théorie, ou le modèle, suppose un système de croyances qu'ils finissent par distiller dans les cerveaux des agents économiques, lesquels ajustent alors le monde à leurs croyances, donc à la théorie. La théorie n'a plus besoin d'être vraie, il suffit qu'elle soit pensée comme vraie par les acteurs, pour que ses hypothèses soient " réalisées " par ces acteurs dans le monde réel. C'est l'explication souvent fournie des bulles financières qui ont suivi le double développement des marchés d'options et de la théorie de Black, Scholes et Merton ${ }^{6}$. Michel Callon s'intéresse davantage aux cas ou le logos d'une théorie transforme le monde réel au point de le

$4 \quad$ Michel Callon, The Laws of the Markets, Londres, Blackwell, 1998 et "Performative Economics », dans Donald MacKenzie, Fabian Muniesa et Lucia Siu (dir.), Do Economists Make Markets, Princeton, Princeton University Press, 2007, p. 311-357.

5 Michel Armatte, Histoire du Modèle linéaire. Formes et usages en Statistique et en Économétrie jusquien 1945, Thèse de doctorat, EHESS, sous la dir. de Jacques Mairesse, 1995.

6 Donald MacKenzie, Fabian Muniesa et Lucia Siu, Do Economists Make Markets?, Princeton, Princeton University Press, 2007. 
rendre compatible avec elle. Dans sa reformulation de $2004^{7}$, il insiste sur le fait que ces cas extrêmes ne sont à ranger dans la catégorie de la performativité que pour autant que l'on puisse mettre en évidence des agencements sociotechniques qui relaient concrètement ces relations d'autorité ou au contraire d'automaticité par convention ${ }^{8}$, persuasion, et croyance de l'inscription dans le réel, le terme d'agencement désignant les dispositifs sociaux et techniques de toutes sortes qui vont effectuer la transformation conforme du monde réel. Ces "agencements", ou " dispositifs ", sont des modèles, des abaques, des logiciels, mais aussi des normes, des règlements, des droits, des lois et des institutions. Et ce que l'on prend pour une confrontation des idées est bien souvent principalement une confrontation entre des agencements concurrents.

Appliquée à l'économie, cette "performativité " des sciences modernes nous oblige à considérer simultanément les aspects académiques de cette science (objets, outils cognitifs, principes, lois), les aspects technologiques et sociaux qui en font une ingénierie (dispositifs de mesure et d'évaluation, dispositifs de régulation par incitation ou règlements, institutions), ainsi que les aspects politiques qui sont sous-tendus par le mode de partage social de l'expertise technique, plus ou moins démocratique, qui caractérise l'articulation entre science et société. L'économie pure, que Callon nomme "économie confinée ", offre une vision beaucoup trop restreinte, et c'est une science économique au sens large, in the wild, " mélangée avec l'ingénierie, les sciences de la vie et celles du management ", plus hétérogène mais plus puissante, qu'il faut considérer. Cette idée d'une science élargie à son

7 Michel Callon, "The Role of Hybrid Communities and Socio-Technical Arrangements in the Participatory Design ", Journal of the Center for Information Studies, vol. 5, no 3, 2004, p. 3-10.

8 Ces conventions ne sont pas arbitraires. Pour nous comme pour les économistes des conventions ou de la régulation, une convention sociale est construite par des mouvements d'opinion, des projets politiques, par le débat public ou médiatique et par les luttes sociales, suite à des événements bien définis, situés géographiquement et historiquement. Or, des événements contingents historiquement ou territorialement ne sont ni hasardeux ni arbitraires. 
ingénierie et à la gestion de ses innovations n'est pas nouvelle, on la trouve évidemment chez les économistes ingénieurs X-Pont qui furent les élèves de Colson, puis dans la génération des militants de X-Crise. Aux États-unis on la trouve chez les inventeurs de l'idée de technocratie, chez les premiers économètres qui se voient comme des "ingénieurs sociaux ". Les travaux historiques ou sociologiques de François Etner ${ }^{9}$ et François Vatin ${ }^{10}$ en France, et de Thomas P. Hugues ${ }^{11}$ et Theodore M. Porter $^{12}$ dans le monde anglo-saxon ont éclairé ce rôle des ingénieurs, mais le plus souvent ils ne l'ont fait que du point de vue d'un apport aux idées ou méthodes économiques sans relever ce que les mêmes acteurs avaient transformé dans le monde " réel ».

Cependant il ne faudrait pas restreindre la proposition «la science économique est une ingénierie " au seul cas bien français des ingénieurs économistes sortant d'une grande école, que ce soit celui de Jules Dupuit travaillant la notion de surplus et inaugurant certaines méthodes de calcul économique, ou les grands commis de l'État, qui un siècle plus tard furent engagés, comme Marcel Boiteux ou Pierre Massé, dans des travaux de recherche opérationnelle pour optimiser des implantations industrielles ou établir des tarifications de services publics. L'exemple de Pierre Massé ${ }^{13}$, ingénieur électricien, découvrant que la gestion des barrages mettait en jeu les deux incertitudes futures de la pluviométrie et de la demande d'électricité nécessitait une pro-

9 François Etner, Histoire du calcul économique en France, Paris, Economica, 1987.

10 François Vatin, Le travail, sciences et société, Université de Bruxelles, 1999 et Économie des forces et production d'utilité. La pensée gestionnaire des ingénieurs des ponts (1831-1891). Édition de la thèse de Bernard Grall (1962-1997), Rennes, Presses universitaires de Rennes, 2004.

11 Wiebe Bijker, Thomas P. Hughes et Trevor Pinch (dir.), The Social Construction of Technological Systems: New Directions in the Sociology and History of Technology, Cambridge (MAP), M.I.T. Press, 1987.

12 Theodore M. Porter, Trust in Numbers. The Pursuit of Objectivity in Science and Public Life, Princeton, Princeton University Press, 1995.

13 Pierre Masse, "Application des probabilités en chaîne à l'hydrologie statistique et au jeu des réservoirs ", Journal de la Société de Statistique de Paris, 1944 , p. 204-219. 
grammation dynamique particulière avait développé les mathématiques d'une telle programmation (avant Bellman à qui on l'attribue) mais, par une analogie puissante sur la notion de réserve, il en avait aussi dérivé une méthode pour problématiser et modéliser le choix entre consommation immédiate et épargne dans un cadre macroéconomique. Commissaire au Plan dans les années 1960, il avait alors mobilisé les mêmes outils que ceux de l'ingénieur pour construire le futur de notre économie.

\section{Exemple de la finance}

De nombreux exemples ont été mobilisés par Callon ou par MacKenzie pour illustrer la performativité des sciences économiques. Mais le plus convaincant est sans nul doute celui de la finance, une branche de l'économie en voie d'autonomisation, du fait même de sa transformation en industrie et de sa conquête de tous les autres marchés. Il n'y a guère de cas aussi exemplaire que l'univers de la finance pour se convaincre du lien fort qui existe entre l'activité académique et l'activité industrielle. Déjà Fabian Muniesa ${ }^{14}$ avait montré comment le marché efficient rêvé par les économistes (Walras en particulier) s'était incarné dans des dispositifs nombreux mis en place lors de l'informatisation de la Bourse de Paris. L'opération révélait toutes les interventions nécessaires à la bonne marche de la Bourse, sans distorsions et effets pervers, au plus près du marché parfait pensé par les économistes.

Au delà des travaux du précurseur que fut Jules Régnault à la fin du XIX ${ }^{\mathrm{e}}$ siècle, la théorie boursière a été établie par Bachelier sur les bases d'un calcul des probabilités classique (loi normale, processus stationnaire brownien...). La théorie des mouvements de cours de Bourse n'a pas supplanté les pratiques professionnelles de l'analyse technique, chartiste ou fondamentaliste, toutes attachées à l'idée de prévisibilité, mais elle les a dominées puis s'est transformée en une ingénierie de la gestion de portefeuille

$14 \quad$ Fabian Muniesa, «Un robot walrasien. Cotation électronique et justesse de la découverte du prix ", Politix, vol. 13, no 52, 2000, p. 121-154 et Des marchés comme algorithmes. Sociologie de la cotation électronique à la Bourse de Paris, Thèse de Doctorat, Paris, École des Mines, 2003. 
dès les années 1950 avec la théorie du portefeuille de Markovitz, qui doit plus au modèle linéaire des économètres de la Cowles Commission qu'à Bachelier, et qui forme la base du modèle CAPM (MEDAF en français), vraiment opérationnel grâce à la simplification de Sharpe en 1964 qui introduit le portefeuille de marché. Couplée à la notion d'efficience des marchés due à Fama $^{15}$ selon laquelle les prix reflètent toute l'information disponible, cette approche ouvrait la voie dans les officines à une gestion passive et indicielle des portefeuilles, confortée par la législation américaine (ERISA), par la multiplication des indices boursiers, et bien sûr par l'informatisation des salles de marché. Aujourd'hui, après la directive européenne MIF (Marché d'instrument financier), la libéralisation du marché boursier a conduit à une multiplication des plateformes boursières, à une automatisation totale des opérations - le " trading algorithmique " - et à une grande opacité de ces opérations $(44 \%$ des transactions sont invisibles selon le Président de l'Autorité des marchés financiers ${ }^{16}$ ).

Après celui de Chicago en 1973, la vague des marchés de dérivés qui se créent à Amsterdam (1978), New York (1979), Londres (1979, 1982), Tokyo (1985) ou encore Paris (1986, 1987) représente une innovation qui permet de se protéger des volatilités de prix de matières premières, des cours boursiers et des risques de change ou de crédit, en s'appuyant sur des modèles théoriques à forte composante mathématique (intégrales stochastiques de Wiener et d'Ito) permettant de gérer ces risques par un portefeuille de couverture établi dynamiquement grâce à la formule de Black et Scholes (1973). Tout produit financier nouveau dérive le plus souvent directement d'une recherche mathématique, et fait l'objet d'un suivi mathématique poussé (et complété par des simulations) pour établir son pricing et sa méthode de couverture. La finance est ainsi devenue l'une des branches de la science économique la plus formalisée et la plus

15 Eugene Fama, «The Behavior of Stock Market Prices ", Journal of Business, $n^{\circ}$ 38, janvier 1965, p. 34-105 et « Efficient Capital Market: II », Journal of Finance, 25 décembre 1970, p. 338-417.

16 «Entretien avec Jean-Pierre Jouyet », Le Monde, 9/12/2010. 
innovatrice. Mais l'économie financière n'est pas seulement une spécialité de diplôme universitaire qui vise à profiter d'un effet d'aubaine, puis à s'autonomiser comme discipline propre; elle est devenue une ingénierie qui envahit les banques et reconfigure totalement le marché financier - ses produits, des acteurs, ses règles - avec l'aide de ses modèles mathématiques et de leur déclinaison informatique. Les faillites grandioses de hedge funds comme le LTCM en 1998 (un ans après que deux de ses fondateurs Scholes et Merton aient eu le prix Nobel pour leur modèle d'évaluation d'options) ou de sociétés accusées d'avoir falsifié leur valeur boursière comme Enron en 2001, ont fait découvrir au monde entier la puissance et la fragilité de château de carte de ce qui n'est plus une invention, plus même une innovation, mais déjà une industrie. Celle-ci ayant mobilisé totalement d'anciens et de nouveaux acteurs dans une logique sociotechnique explosive qui n’avait été clairement anticipée ni par les inventeurs de ces produits, ni par les ingénieurs mathématiciens qui en ont optimisé la gestion, ni par les banquiers et spéculateurs qui s'en sont emparés, ni par les citoyens qui découvraient, effarés, un monde qu'ils ignoraient.

Que nous apprend encore l'actualité récente de la crise financière des subprimes? La plupart des analystes ont bien cartographié aujourd'hui ses épisodes et ses caractéristiques, et ont largement discuté ses fondements qui se trouvent autant dans les effets incontrôlables d'une ingénierie financière ayant permis tous les débordements d'une saine gestion des risques, que dans les bases micro et macro-économiques d'un régime post-fordiste ultralibéral ayant mis les logiques industrielles sous la dépendance des marchés financiers, puis fait de même des logiques sociales en construisant sa prospérité sur l'endettement croissant des ménages américains

17 Voir Michel Aglietta et Antoine Rebérioux, Dérives du capitalisme financier, Paris, Albin Michel, 2004; Patrick Artus et al., La crise des subprimes, Rapport du Conseil d'Analyse Économique, Paris, La Documentation française, 2008; Frédéric Lordon Jusqu'à quand? Pour en finir avec les crises financières, Paris, Raison d'Agir, 2008; Paul Jorion, La crise. Des subprimes au séisme financier planétaire, Paris, Fayard, 2008; Jacques Sapir, "Une décade prodigieuse. 
L'histoire récente de la finance, révisée par la crise qu'elle vient de subir, nous invite à réévaluer la théorie des marchés efficients, postulant une convergence des intérêts individuels et de l'intérêt général, à réévaluer l'idée de valeur fondamentale et les modèles probabilistes ayant servi au calcul des risques. Elle nous révèle aussi le rôle de cette théorie dans la construction même des marchés. On a bien passé des ordres sur des marchés en faisant comme si les marchés étaient efficients et produisaient une fair value, jusqu'à ce que la crise prouve le contraire. André Orléan ${ }^{18}$ soutient même que le marché financier qui entre dans une bulle spéculative n'est plus un marché au sens de la théorie classique puisque la montée des prix ne provoque pas une baisse de la demande mais son augmentation. On a bien compris aussi que les modèles probabilistes enrôlés dans l'évaluation et la gestion des risques avaient des hypothèses d'indépendance et de normalité qui ne correspondaient pas aux risques réels pris par les acteurs.

Toute analyse en profondeur de la crise financière oblige à dépasser ce point de vue théorique pour prendre en compte les aspects organisationnels : comment fonctionnait la titrisation, quelles ont été les dérégulations qui ont permis aux banques de prendre tous les risques, quelles ont été les faiblesses congénitales des agences de notation, sur quelles caractéristiques non permanentes du marché immobilier américain ces prises de risque se sont elles appuyées, quels rôles ont eu de nouvelles pratiques comptables comme le mark-to-market et le mark-to-model? Le phénomène de la bulle et de son éclatement, qui faillit emporter

La crise financière entre temps court et temps long ", Revue de la régulation, $2^{\text {e }}$ semestre 2008, sur le site : http://regulation.revues.org/document 4032 . html, consulté en décembre 2010; Michel Armatte, "Crise financière : modèles du risque et risque de modèle ", Mouvement, 2009, sur le site www. mouvements.info/crise-financiere-modeles-du-risque.htm consulté en janvier 2011; André Orléan, De l'euphorie à la panique : penser la crise financière, Paris, Éditions de la rue d'Ulm, coll. « opuscule du cepremap » 2009.

André Orléan, De l'euphorie à la panique..., op. cit.

19 Christian Walter et Eric Briand (dir.), Critique de la valeur fondamentale, Springer, 2007; Christian Walter et Michel de Pracontal, Le virus B. Crise financière et mathématiques, Paris, Le Seuil, 2009. 
tout le système bancaire, n'est perceptible dans sa globalité que si l'on prend en compte les formes industrielles et institutionnelles prises par ces innovations, les régulations mises en place, les savoir-faire et les règles mobilisés, bref l'ingénierie financière. Pour autant que la finance relève des interactions d'acteurs, de leurs croyances et de leurs modalités de décision, cette analyse doit retrouver une pluralité d'hypothèses théoriques ${ }^{20}$, en allant chercher des savoirs de type sociologiques, anthropologiques, psychologiques, professionnels et institutionnels sur les manières de faire son métier dans chaque lieu d'intervention du secteur financier - savoirs longtemps tenus pour anecdotiques mais seuls à même de nous dire ce qu'est la science financière en action.

\section{Les économistes dans la ville}

Il ne faudrait pas déduire de ce qui précède que « l'effet performatif de la théorie économique " n'existe que dans le domaine de la finance. Il est simplement plus visible et surtout il opère à des échelles spatiales mondiales, dans des conditions d'automaticité et avec des si petits ${ }^{21}$ que les effets y sont foudroyants. Notre propos est cependant plus large et consiste à reconnaitre que la science économique dans toutes ses composantes peut, aujourd'hui plus que jamais, être assimilée à une ingénierie. Cela signifie d'abord tout simplement que la sphère académique au sens restreint (enseignants et chercheurs) ne constitue pas $10 \%$ des débouchés des étudiants en économie et gestion. Il est facile de montrer que la grande majorité des économistes diplômés comme tels occupent des postes de conseillers, de chargés d'études, d'experts dans des entreprises, ou auprès d'organismes, et qu'ils ont dans ces postes des tâches de design, d'évaluation, de contrôle de dispositifs traduisant des politiques sectorielles, ter-

\footnotetext{
20 On pense par exemple aux hypothèses d'autoréférencialité et d'instabilité des marchés financiers d'André Orléan qui résultent d'une observation réelle de leur fonctionnement.

21 Le trading à haute fréquence permet des gains importants en passant des ordres automatiques dans des fenêtres de temps de l'ordre de la milliseconde. Voir "High Speed Trading " et "Flash Ordres ", Le Monde du 9 décembre 2010 .
} 
ritoriales, nationales ou internationales, publiques ou privées. Même dans le cas d'un profil d'universitaire, on trouve qu'il est associé dans 8 cas sur 10 à d'autres fonctions que l'enseignement et la recherche, qui engagent ces académiques dans des secteurs comme les banques et assurances, les organismes internationaux (BIRD, FMI, Banques centrales, OCDE), les groupes industriels, le journalisme et les médias, les mandats politiques, les agences de l'État (CAE, CAS, OFCE...), les administrations (Trésor, Budget...), les Associations et ONG... ${ }^{22}$

La grande majorité des économistes se trouve enrôlée par les différents corps d'État, les agences, les conseils à différents niveaux. Brefs ils ne sont pas que des théoriciens se contentant de lire, échanger et publier sous le contrôle de leurs pairs. En d'autres termes les économistes ne sont pas enfermés dans la tour d'ivoire de leurs tâches académiques et épistémologiques, se contentant de débattre entre eux des paradigmes (Kuhn) ou des programmes de recherche (Lakatos) qui sont en concurrence pour interpréter le monde économique. Ils ne sont pas que des observateurs avisés du bruissement des marchés économiques. Ils en sont des acteurs privilégiés, tous plus ou moins engagés dans la cité, à construire, adapter et défendre tel ou tel dispositif. Bien sûr ils ne sont pas forcément les entrepreneurs constructeurs de ces dispositifs, mais ils en sont les architectes, les designers, et parfois les réparateurs ou les pompiers.

Ce constat n'est pas original, mais ce qui l'est davantage c'est de considérer que ces tâches d'architectes-entrepreneurs-adaptateurs-dépanneurs font partie au même titre que la recherche théorique de la science économique. De ce fait, dans une tradition STS (sciences-techniques et société) nous ne parlerons plus de science mais de techno-sciences. En effet, depuis le début du XX siècle, il est devenu impossible de séparer sciences et techniques. Isoler la première en disant que les innovations techniques et économiques ne sont que des constructions postérieures aux

$22 \quad$ Voir par exemple les notices biographiques des 160 intervenants aux Journées de l'économie, ou encore les annuaires de sociétés savantes comme l'AFSE ou la SEP. 
découvertes scientifiques n'est pas conforme à ce que les historiens et sociologues des sciences ont observé. Même en considérant qu'il existe toujours des lieux de développement de la science pure comme la physique théorique testée sur les anneaux du CERN, on doit reconnaître qu' une bonne partie de la science se développe parce qu'elle est tirée par les applications qui en sont perceptibles dès le début, quand ce n'est pas la recherche appliquée qui elle-même produit des résultats théoriques. En tous cas on doit prendre en compte les deux processus d'une accumulation de la connaissance centrée sur le cœur de la discipline ou produite à sa périphérie.

\section{Histoire économique et science studies}

Une fois admise l'idée que toute question relative aux rapports entre science économique et société oblige à prendre en compte la science économique comme ingénierie, il faut discuter le point de savoir quelle est la discipline la mieux placée pour cela. $A$ priori, pourquoi ne serait-ce point la science économique ellemême qui traite de ses rapports avec la société? Toutes les disciplines ont produit des discours sur leur légitimité à parler comme elles le font des objets qui sont les leurs. La question s'est par exemple posée aux mathématiciens qui ont longtemps rejeté l'idée que des non-mathématiciens puissent saisir tous les enjeux de leur discipline. La philosophie des mathématiques, l'histoire des mathématiques et la didactique des mathématiques sont ainsi longtemps restées fermées aux non-mathématiciens. On en est un peu revenu dans les dernières décennies dès lors que l'on s'intéresse au rôle des mathématiciens dans la société. S'il y a une certaine inconscience à prétendre réaliser des études critiques d'une science sans en connaître intimement les méthodes et concepts - soit par formation, soit par positionnement anthropologique -, il y a symétriquement un risque au moins aussi important à penser que cette position réflexive puisse être tenue uniquement de l'intérieur de la discipline.

Il en est de même pour la science économique, laquelle comprend parmi ses activités académiques deux traditions d'étu- 
des réflexives : l'histoire de la pensée économique et l'épistémologie économique. Mais peut-on se satisfaire de leurs recherches? En effet, comme nous l'avons vu avec l'épisode de la critique " autiste », ces approches réflexives sont sous-dimensionnées voire souvent absentes des formations traditionnelles. L'épistémologie, l'histoire de la pensée, et plus encore l'histoire des faits ont quasiment disparu des cursus de Licence et Maîtrises pour se retrouver aujourd'hui, réforme LMD aidant, confinées dans un petit nombre de filières de master spécialisées à petits effectifs. Au-delà de ces arguments portant sur l'organisation des études, il y a aussi un argument plus profond : les économistes académiques ont trop souvent surestimé la théorie, les principes et les modes de raisonnement, qui forment selon eux le cœur de leur discipline, ce qui les a conduits à sous estimer les activités de transformation du monde réel que leurs innovations ont suscitées, planifiées et organisées. Peut-être ne sont-ils pas les mieux placés pour parler de leur rôle dans la société!

Les économistes sont pour beaucoup d'entre eux juge et partie de l'adéquation et de l'efficacité des politiques mises en œuvres. La crise économique que nous vivons aujourd'hui à la suite du krach financier des prêts subprime a produit une crise de confiance dans le système financier et tout particulièrement vis-à-vis des banques qui ont joué avec le risque. Mais elle a produit aussi une certaine méfiance vis-à-vis des économistes qui ont accompagné la vague des innovations financières par un discours ultralibéral immodéré et parfois messianique. Il faut donc reconstruire non seulement le marché financier mais aussi l'image publique du rôle des acteurs économiques.

Tenir simultanément ces différents aspects - cognitifs, techniques, sociaux et politiques - dans une analyse de la science économique telle qu'elle se fait ${ }^{23}$ et telle qu'elle agit n'est donc pas une tradition de l'enseignement et de la recherche économique, et peut même s'avérer incompatible avec le non-réalisme des principes adoptés par le main stream néo-classique. À considérer

23 Allusion à Bruno Latour (dir.), La science telle qu'elle se fait : anthologie de la sociologie des Sciences de langue anglaise, Paris, Pandore, 1982. 
des entités abstraites - agents, homo ceconomicus - qui se rencontrent sur des marchés efficients et équilibrés, on ne voit pas ce que ces analyses pourraient apporter comme vision réflexive et sociologique sur leur performativité, leur efficacité ou leurs effets pervers dans la société. La réflexion des spécialistes de l'histoire de la pensée qui, parfois, enfilent les idées comme des perles dont ils font des colliers et des catalogues, ne prend guère en compte le fait que cette pensée est dans des rapports étroits avec les conditions techniques, politiques et sociales d'une économie située ici et maintenant. Les « hétérodoxes » - institutionnalistes, conventionnalistes, régulationnistes - ont certes fourni des cadres importants pour penser ces liens entre la science économique et son objet « réel " mais ils ont fortement réduit le statut des outils mathématiques et statistiques à un rôle instrumental, et ils ont longtemps ignoré les acquis des études socio-historiques sur les sciences.

Dernier argument : l'étude des sciences est a priori une discipline qui ne saurait être confondue avec la discipline objet qu'elle se choisit. L'histoire sociale des mathématiques n'est pas mathématique et ne produit pas de théorèmes. L'histoire de la médecine ne soigne personne. L'étude des sciences a, ou doit avoir, sa propre méthode, distincte de celle de la discipline cible. Elle bénéficie aujourd'hui d'une série de discussions approfondies menées sur les principes de symétrie, de réflexivité, d'explication rationnelle $e^{24}$, d'une tradition d'analyse des pratiques de laboratoire, d'une attention aux stratégies d'acteurs individuels ou institutionnels, d'un agnosticisme salutaire quant à la vérité ou l'efficacité des théories, qui lui permettent de décortiquer les éléments d'une controverse ou d'une tentative de traduction imposée $^{25}$, donc de construire une distance avec son objet.

\footnotetext{
24 Le programme fort de la socio-logique de David Bloor, Sociologie de la logique ou les limites de l'épistémologie, trad. Ebnöther, Paris, Pandore, 1982 [1976].

25 Traduction au sens de Callon. Voir Michel Callon, "Éléments pour une sociologie de la traduction. La domestication des coquilles Saint-Jacques et des marins-pêcheurs dans la baie de Saint-Brieuc ", L’Année Sociologique, $\mathrm{n}^{\circ} 36,1986$, p. 169-208 et de la théorie des acteurs-réseaux, c'est-à-dire le déplacement des objectifs, trajectoires, instruments et alliances d'un acteur pour les faire servir à un autre acteur.
} 
C'est pourquoi nous pensons qu'il est intéressant de balancer la vision critique faite de l'intérieur de la discipline économique, par des éléments d'analyse exogène empruntés à l'histoire des sciences, et plus particulièrement à l'école des sciences studies.

Cette école, dont l'origine se situe dans l'École de Bath en Angleterre, et qui a bénéficié en France des travaux de Bruno Latour, Michel Callon, Dominique Pestre et quelques autres, a mené un grand nombre d'études empiriques, dans de nombreux domaines et pour des périodes anciennes, modernes ou postmodernes. Héritant des acquis de la philosophie de la connaissance sur l'analyse logique des théories, façon Cercle de Vienne puis Popper, avant le tournant sémantique des années 1960, puis le tournant cognitiviste des années 1990, elle s'est aussi appuyée sur les travaux de l'histoire classiques des sciences. Petit à petit émancipée de la tutelle des philosophes, et se tournant depuis les travaux de Kuhn vers la sociologie et les sciences politiques pour rendre compte de paradigmes et de communautés historiquement située, l'école des science studies a renouvelé totalement l'historiographie de la période moderne et s'est spécialisée dans l'étude des controverses. Non sans susciter elle-même des controverses sur sa méthode dont la plus célèbre fut l'affaire Sokal ${ }^{26}$. Sortie renforcée de ces épreuves, elle s'est montrée très efficace pour traiter de l'articulation entre science technique et société (STS). En n’isolant jamais les caractéristiques cognitives des caractéristiques sociales dans la pratique scientifique, en acceptant d'entrer dans le laboratoire, de suivre ses protocoles, ses raisonnements, ses preuves, mais en acceptant aussi d'en sortir pour voir comment le savant et son laboratoire se projettent dans la société politique et marchande, les science studies offrent des exemples et des outils que nous pouvons mobiliser pour traiter de la science économi-

26 Par exemple l'épisode de la "guerre des sciences » déclenchée après l'affaire Sokal de 1996 : au-delà du canular de Sokal et Bricmont qui visait à déqualifier toute sociologie des sciences, on peut considérer que cette affaire est une réaction visant à revenir au premier modèle d'expertise, et à nier toute possibilité d'une évaluation extérieure (philosophique, historique, sociologique) des pratiques scientifiques. Il est dès lors facile de retourner contre eux l'argument obscurantiste des sokaliens. Voir Baudoin Jurdant (dir.), Impostures scientifiques. Les malentendus de l'affaire Sokal, Paris, La Découverte 1998. 
que dans la cité.

Certes les études sur les sciences ont trop longtemps privilégié les sciences physiques et naturelles prises comme modèle épistémologique, aux dépens des sciences sociales accusées de produire des énoncés non vérifiables et non réfutables, et trop facilement soumis aux pressions idéologiques et aux intérêts de certains groupes sociaux ou du pouvoir politique. Mais l'économie et la gestion, qui sont au cœur des articulations entre science et société, redeviennent un objet d'intérêt incontournable dès lors que l'on traite de technosciences ${ }^{27}$ au sens large, car les processus sociaux d'innovation et de transformation sociale qui sont en jeux mêlent, à diverses échelles, des savoirs et des croyances appartenant aux mondes artificiellement séparés des sciences de la nature, des sciences sociales, de la gestion politique et quotidienne. Nous pouvons donc utilement confronter les travaux des historiens de l'économie avec ceux des science studies, à commencer par la question des régimes de savoir.

\section{Régimes de savoir}

Pour rendre compte de l'ingénierie économique, il est donc intéressant de la situer dans l'histoire plus générale des technosciences. Mais cette caractérisation est-elle universelle et atemporelle? Certains historiens soutiennent qu'il y eut toujours, depuis les physiocrates, une certaine implication des économistes dans la cité; ils font remarquer que les débats sur les poor laws ou sur les corn laws ont impliqué des économistes de renom, et que les ingénieurs de l'ancien régime qui ont construit nos ponts nos routes et nos canaux ont défendu leurs projets en mobilisant leur science du calcul économique. L'économie politique du XIX ${ }^{\mathrm{e}}$ siècle était caractérisée par un lobbying forcené des libéraux dans les différentes arènes du pouvoir, à commencer par la presse, à finir par la Chambre des députés. Mais il est clair que les maniè-

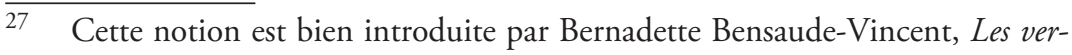
tiges de la techno-science. Façonner le monde atome par atome, Paris, La Découverte, 2009, qui en donne trois caractéristiques principales : «l'accent mis sur les opérations, l'abolition du fossé entre l'homme et la nature; enfin l'individuation des objets ». 
res d'intervenir au sein des processus de décision et de s'impliquer dans l'élaboration même des lois, des règlements, des institutions n'ont pris ni la même ampleur ni la même forme au cours des deux derniers siècles. En particulier parce que les rôles des différentes institutions (l'État, le marché, les universités, les entreprises) et des différents outillages (philosophique, mathématique, informatique) changent radicalement.

C'est ici qu'il faut alors introduire la notion de régime de savoirs. Une notion qui ne vient pas de la discipline économique elle-même mais de l'histoire des sciences en général, et plus particulièrement de l'école des science studies et de la nébuleuse STS. Dominique Pestre définit un régime de savoirs comme " un assemblage d'institutions et de croyances, de pratiques et de régulations politiques et économiques, qui délimitent la place et le mode d'être des sciences dans la société ${ }^{28}$ ". La thèse de Pestre est que "les scientifiques ont été depuis longtemps dans des réseaux excédant largement les seules structures académiques et universitaires " et que ceux qui verraient une rupture radicale entre deux modes de production des savoirs ${ }^{29}$ dominés respectivement par la science pure et la science appliquée ne prennent pas en compte le fait historique que la science qui se met en place à l'époque moderne "a toujours été du plus haut intérêt pour les pouvoirs politiques, économiques et militaires ", et que cette polarisation ne résiste pas à l'examen historique. En fait l'histoire des sciences dans ses rapports avec la société permet de distinguer un plus grand nombre de ces régimes qui se succèdent et parfois coexistent, mettant en œuvre une grande variété de pratiques, de valeurs, de normes, d'institutions et de gouvernement des sciences tissant un lien variable avec le gouvernement des entreprises, des États et des individus, et de leurs diverses socia-

28 Ces régimes de savoir sont, par exemple, l'objet du séminaire " penser les sciences au XX $\mathrm{XX}^{\mathrm{e}}$ siècle " tenu au centre Koyré depuis 2001 et repris par Dominique Pestre dans Science, argent et politique. Un essai d'interprétation, Paris, INRA, 2003, (voir p. 36).

29 Michaël Gibbons et al. (dir.), The New Production of Knowledge: The Dynamics of Science and Research in Contemporary Countries, London and Thousand Oaks, SAGE, 1994. 
bilité (familles, ménages, associations, etc.). Le régime qui naît avec la science moderne au XVII ${ }^{\mathrm{e}}$ siècle en Angleterre et en France est assez largement dominé par une reconstruction des modes de savoir et des degrés de croyance sur la base de la logique (par exemple celle de Port Royal, celle des philosophes comme Hobbes et Locke), de la constitution et de la circulation des preuves expérimentales et des observations de la nature (afin de dépasser la cacophonie des témoignages et des mesures instrumentales), avec un contrôle et une légitimation de ces modes d'opérer par les Académies et l'État ingénieur au sens colbertien. Et ce régime des sciences s'articule parfaitement avec la naissance un peu partout de l'État-nation. Un second régime accompagne la révolution politique (république et démocratie) et la révolution industrielle et associe à la science " pure " une ingénierie technique et sociale, Saint Simonienne puis positiviste, très présente en France à travers les grands corps de l'État. Le régime qui s'installe à la fin du XIX ${ }^{\mathrm{e}}$ siècle, et surtout après la première guerre mondiale, voit la science devenir " un élément central des dispositifs d'innovation et de rationalisation " avec un rôle tout à fait nouveau pris par l'État, d'abord dans son rôle d'État-Providence assurant la protection des travailleurs et entrepreneurs dans un rapport de classe difficile, puis après les crises financières et économiques des années 1930, dans son rôle d'un Etat-régulateur bientôt caractérisé de keynésien ${ }^{30}$. Les techno-sciences deviennent alors un enjeu pour l'État qui devient lui-même un entrepreneur scientifique, propriétaire de près de $50 \%$ des moyens de productions en France dans l'immédiat après guerre. Une des caractéristiques essentielles de ce régime est un quadruple mouvement de réductionnisme (qu'illustrent aussi bien la physique et la biologie que l'économie), de quantification, de formalisation - en particulier mathématique - de simulation des théories scientifiques, et enfin, de déclinaisons pratiques des savoirs académiques, qui voit les univers savants et techniciens s'articuler fortement en

$30 \quad$ Alain Desrosières, "L'État, le marché et les statistiques : cinq façons d'agir sur l'économie ", Courrier des Statistiques, n ${ }^{\text {os }} 95-96$, décembre 2000, p. 3-10, et Problèmes économiques, n 2725, 29 août 2001, p. 26-32. 
un paradigme techno-scientifique. Les sciences "pures " profitent des travaux d'ingénieurs et, vice versa, les travaux des ingénieurs sont de plus en plus adossés aux découvertes des sciences pures, même si cet échange est masqué par ce que Pestre appelle "le hold up intellectuel et rhétorique des clercs » qui maintient le mythe, rarement déconstruit, d'une science pure, matrice de tous les progrès ${ }^{31}$.

Les sciences économiques s'inscrivent parfaitement dans ce régime de guerre froide et de " glorieuse " croissance, qui voit s'affirmer à la fois la nouvelle alliance de la recherche avec le complexe militaro industriel (rapport de Vanevar Busch et discours d'Eisenhower), la domination du dollar américain sur l'économie (accords de Bretton Woods) et celle des nouveaux think tanks et universités américaines sur la science économique. La recherche opérationnelle, la théorie des jeux, l'économétrie développées pendant la guerre à la Cowles Commission et à la Rand Corporation deviennent après guerre les nouveaux outils standards des économistes engagés dans des travaux importants de régulation et de prévision qui relèvent bien d'une ingénierie économique profitant de l'essor des mathématiques appliquées, de l'optimisation, de la planification, de grâce à l'ordinateur et aussi à la notion de modélisation qui s'impose partout en économie mais évolue du calcul à la simulation et de la gestion automatisée $^{32}$. Ce régime, que les macro-économistes régulationnistes nomment fordiste, va durer jusqu'au milieu des années 1970 : le choc pétrolier et les premières limites à la croissance et la consommation de masse, puis le virage libéral de Thatcher et

31 "Gardez-vous de la pureté, c’est le vitriol de l'âme " écrit Tournier dans Vendredi ou les limbes du Pacifique, repris par Brunot Latour dans Irréductions (Les microbes : guerre et paix, suivi de Irréductions, Paris, Métaillé, 1984). Comme réaction à ce délire de pureté, voir par exemple Didier Nordon, Les mathématiques pures n'existent pas, Paris, Actes Sud 1993 [1981].

32 Voir E. Roy Weintraub, How Economics Became a Mathematical Science, Durham and London, Duke University Press, 2002; Michel Armatte et Amy Dahan, "Modèles et modélisations (1950-2000) : nouvelles pratiques, nouveaux enjeux ", Revue d'Histoire des Sciences, vol. 57, n 2, 2004, p. 245305; Michel Armatte, La science économique comme ingénierie. op.cit., chap. 7 et 8 . 
Reagan dans le cadre du " consensus de Washington ", et enfin l'irruption d'un capitalisme financier favorisé par de nouveaux régimes de propriété du capital (les actionnaires prennent le pas sur les entrepreneurs et alignent la rentabilité industrielle sur celle des marchés financiers) et de la propriété intellectuelle (extension du domaine des brevets à toute forme d'innovation y compris sur le vivant, entrée du capital-risque dans le domaine de la recherche), marquent du point de vue socio-économique le début d'un nouveau régime d'articulation entre science et industrie, et tandis que l'insistance nouvelle sur les fondements micro de la macro, sur les anticipations rationnelles, la vogue des modélisations de type MEGC et MSEG ${ }^{33}$, la modélisation intégrée du changement climatique, la balance entre risque calculé et principe de précaution, en traduisent le tournant méthodologique dans le domaine économique.

\section{Régimes d'expertise}

À chacun des régimes de savoir que nous avons évoqués correspond un certain type de rapport entre sciences et politique, et donc une certaine forme historique de l'expertise. La science de la seconde moitié du XIX ${ }^{\mathrm{e}}$ siècle ne concevait guère d'autre rapport au politique que celui de l'exclusion : la politique comme domaine des idéologies et des puissances d'argent doit être tenue à l'écart du développement d'une science pure, élaborant ses concepts et ses énoncés hors de toute influence, et dont l'évaluation se fait à l'échelle de la compétition internationale dans un marché des idées bien isolé des autres marchés. La sociologie des sciences du début du siècle, aussi bien celle de Merton développant l'idée d'une communauté propre de savants ayant leurs normes et valeurs, que celle d'obédience marxiste (avec des auteurs comme Bernal ou Manheim) a gardé des traces de cette séparation, et d'un modèle d'analyse fondé sur l'asepsie et la non contamination des normes et valeurs de la science par celles de la classe politique. Les guerres de 1970 et de 1914 et les grandes

33 Modèle d'équilibre général calculable, modèle stochastique d'équilibre général. 
crises économiques des années 1890 et 1930 ont modifié profondément ce rapport en instaurant ce que l'on a nommé la technocratie, un mouvement démarré aux États-Unis dans les années 1910 et bien illustré en France par l'épisode X-Crise : il devenait légitime et même hautement souhaitable que scientifiques et ingénieurs puissent s'investir dans des programmes de modernisation de nos moyens militaires et économiques. Ce nouveau modèle d'articulation entre science et politique est dit linéaire parce qu'il suppose que les sciences pures peuvent se prolonger par des sciences appliquées qui elles mêmes peuvent s'illustrer dans des dispositifs techniques rencontrant - quel hasard! - des besoins sociaux. Ce modèle protège encore les scientifiques d'une demande sociale plus directe émanant soit de la programmation industrielle ou militaire, soit des exercices de prévision et de planification, soit des opérations marketing des industriels. Mais les discours des présidents américains des années 1940 et 1950 sont là pour témoigner d'une nouvelle alliance ayant pris le nom de complexe militaro industriel et savant. Que les premiers l'aient emportés sur les derniers compte tenu des conditions particulières de la guerre mondiale puis de la guerre froide est possible; toujours est-il que le modèle linéaire ne rend plus compte de ce qui se passe depuis les années 1970 : les grandes crises, qui sont celles de l'économie mondiale après le choc pétrolier, ou celles des risques nucléaires puis sanitaires (vache folle, grippe aviaire...) et enfin les crises environnementales et climatiques des années 1990, ont induit une perte de confiance envers les productions idéologiques et matérielles des sciences, et pour finir une remise en cause de l'expertise, détruisant le modèle précédent au profit d'une vision délibérément plus politique : il revenait légitimement au politique de formuler des cadrages de la demande d'expertise en plaçant la décision, le principe de précaution, et la gouvernance de la lutte contre les externalités négatives de la croissance au centre voire au démarrage du processus, instrumentalisant l'expertise scientifique pour qu'elle ne réponde pas seulement à la question " où va le monde " mais plutôt à celle-ci : « que faire pour qu'il aille plutôt dans telle 
direction que dans telle autre ». Les travaux de Foucault sur la gouvernementalité aussi bien que ceux des science studies, occupés tout autant de logiques d'acteurs que de logique cognitive, ont constitué un écho et une prise de conscience de ce troisième régime.

La crise de confiance dans les sciences issue de ces risques dits systémiques est aussi une crise de l'expertise en tant que telle dans ces diverses formes connues. La question posée n'est plus comment doit s'articuler le travail de recherche des savants et la demande politique, mais comment faire en sorte que cette articulation ne se caractérise pas par une élimination du citoyen ordinaire dans le processus d'expertise. Comment le réinsérer dans les processus d'évaluation et de décision? Comment concilier expertise et démocratie? Les solutions sont en partie connues : pour démocratiser l'expertise, il faut d'abord diffuser à tous un maximum d'éléments de compréhension des enjeux des problèmes et des solutions mises en place, puis redonner au citoyen une place dans le débat et la décision, en s’appuyant à la fois sur les éléments de la démocratie formelle - la presse, les enquêtes parlementaires, les campagnes électorales - et sur des formes de démocratie participative plus directes, au sein par exemple de " forums citoyens ", ou de " forum hybrides " mélangeant politiques, experts et populations concernées ${ }^{34}$. Mais de l'idée à la mise en œuvre et à son appropriation par les citoyens eux-mêmes il y a encore loin...

\section{Conclusion : faut-il avoir peur de la politique?}

L'expression "les économistes dans la Cité " s'oppose évidemment à l'idée que l'économiste puisse s'en tenir à son rôle académique de production et reproduction d'une connaissance savante dans sa tour d'ivoire ou dans le seul forum constitué de ses pairs. Mais, pour peu que l'on regarde au-delà de cette fonction académique, quelles sont, pour finir, les formes de cette intervention dans la cité? J'en vois trois formes idéales assez distinctes, corres-

34 Michel Callon, Pierre Lascoumes et Yannick Barthes, Agir dans un monde incertain. Essai sur la démocratie technique, Paris, Seuil, 2001. 
pondant à des investissements politiques différents.

La première forme est celle de l'engagement citoyen, par laquelle l'économiste s'engage dans des pratiques sociales et politiques qui peuvent prendre différentes formes : d'une simple participation au débat public avec prise de position dans les différents forums que sont les réunions publiques, les instances syndicales, et les médias de tout type - la presse écrite que les économistes ont l'habitude d'investir, les plateaux de radio et télévision de plus en plus, mais aussi les fameux blogues sur Internet qui se multiplient; mais cela peut se prolonger par un engagement plus formel et plus durable dans des structures syndicales ou politiques, dans un exercice du droit de vote, et éventuellement dans des mandats électifs locaux (commune, département, région) ou nationaux voire européens. Dans ces engagements, l'économiste peut ne rien engager d'autre que son opinion, ses choix et les valeurs qu'il porte. Et son action peut n'avoir rien de commun avec sa formation et ses compétences professionnelles. Comme on le dirait pour un astronome élu dans un conseil municipal. Quel risque y aurait-il d'une quelconque interaction entre sa pratique scientifique et son engagement politique? Aucun bien sûr. Cela est plus difficile cependant pour un économiste tant il y a quelques liens entre économie et gestion publique. Mais cela reste une position de référence dans la déontologie professionnelle. On colle ici au premier modèle de rapport science et politique qui est celui de la non-contamination, de l'indépendance voulue et cultivée entre pratique de la science et engagement politique.

La seconde forme d'engagement dans la cité est beaucoup plus profondément liée à une compétence de spécialiste acquise par la formation et la recherche et correspond à ce que nous avons appelé l'ingénierie économique, soucieuse d'application de connaissance savante à des dispositifs sociaux opérationnels. On pourrait évoquer ici la liste assez longue des disciplines de l'économie appliquée, et des professions ouvertes à l'économiste en dehors de l'enseignement et la recherche : journalisme économique, études économiques, activités de conseil, carrières politi- 
ques... Et le modèle sous-jacent à l'œuvre ici serait celui de la technocratie et de l'expertise linéaire. La fonction dans la recherche déboucherait in fine sur des utilisations de régulation et de transformation sociale, voulues ou non voulues, réussies ou non, voire à des performations de la société à des fins d'adaptation à la théorie économique dont l'effet est plus sournois.

La troisième forme me paraît relever plus spécifiquement de l'expertise concertée et coconstruite avec les politiques, c'est-àdire d'un travail d'évaluation, de conseil et d'innovation qui est une réponse à une demande politique explicitement formulée au sujet d'un problème, d'un enjeu ou d'une politique. Le cas du mathématicien (Poincaré, Borel l'ont fait) exerçant une expertise graphologique auprès des tribunaux est un cas d'école. On peut lui associer le cas de l'économiste chargé d'un rapport sur tel sujet auprès du Ministre concerné, ou bien celui qui se trouve engagé dans le programme d'évaluation du GIEC sur les impacts du changement climatique (groupe II) ou sur les politiques de réduction (groupe III). Ici le troisième modèle d'expertise, caractérisé par le primat de la demande politique, semble s'imposer et fait la différence avec le cas précédent.

Bien sûr cette distinction n'est pas toujours aussi claire. Il peut être difficile de dire si tel ou tel engagement de l'économiste dans la cité est du second type ou du troisième. Mais une telle distinction peut clarifier l'analyse socio-historique aussi bien qu'aider à une prise de position plus engagée de notre part. Car pour finir, faut-il souhaiter ou regretter que l'économiste sorte d'un rôle purement académique? Poser la question de cette façon, c'est, me semble-t-il, s'apprêter à recevoir des réponses positives sur les deux premiers cas, mais beaucoup plus partagées sur le troisième. Tout le monde doit reconnaître facilement que l'économiste a toute latitude pour s'engager dans la cité comme n’importe quel

35 Voir sur ce point Amy Dahan-Dalmedico (dir.), Les modèles du futur, Paris, La Découverte, 2007; Encinas de Munagorri (dir.), Expertise et gouvernance du changement climatique, Paris, LGDJ, 2009; Michel Armatte, La science économique comme ingénierie, op. cit., chap. 11; Jean-Philippe Touffut (dir.), Changement de climat, changement d'économie, Centre Cournot, Albin Michel, 2010. 
citoyen, s'il ne mélange pas ses convictions et ses travaux scientifiques. Tout le monde ou presque peut reconnaître que la science économique a de nombreux prolongements dans l'ingénierie économique, qui assurent d'ailleurs à la discipline une bonne partie de sa reconnaissance sociale. Le progrès des sciences économiques, comme ceux de la physique et de la biologie, sont davantage perceptibles pour le profane à partir de leurs avancées opérationnelles dans des dispositifs techniques que par les progrès enregistrés sur le plan théorique, dont l'évaluation reste une énigme même à l'aune de la falsification poppérienne. Affirmer par contre que la demande politique peut susciter et formater largement un programme de recherche est encore hautement problématique. Même lorsque ce programme émane d'une légitime interrogation selon des procédures que l'on peut qualifier de démocratiques. On l'a vu sur l'exemple climatique : les rapports de synthèse du GIEC établis sur la base de publications des climatologues ont été un moment soupçonnés de ne pas pouvoir être considérés comme scientifiques parce qu'ils étaient réalisés dans le but d'une expertise auprès d'une instance politique mondiale, l'UNFCC, responsable de la négociation politique entre les parties (les États) cherchant à déterminer une politique supranationale de lutte et d'adaptation face au changement climatique. Les arguments échangés à l'occasion de cette controverse ont largement montré que les principes d'une science neutre et indépendante ont semblé pour beaucoup incompatibles avec l'idée d'une expertise répondant à une demande politique, futelle non partisane, fut-elle même guidée par un accord politique réalisé au plus haut niveau de la gouvernance mondiale. Une science pour la décision politique? L'idée n'en est pas encore mure. Elle semble encore trop dangereuse. Elle devrait pourtant s'imposer petit à petit compte tenu des défis de "la société du risque $"$ que nous connaissons depuis quelques décennies et qui pèsent plus sur l'avenir de notre humanité que l'idéal d'une science pour la science.

36 Ulrich Beck, La société du risque. Sur la voie d'une autre modernité, Paris, Aubier, 2001. 
Le vœu que nous pourrions formuler pour conclure comporterait plusieurs volets : d'abord ouvrir les portes et fenêtres de la discipline et y faire rentrer l'air de la cité, c'est-à-dire les problèmes et solutions de la vieille économie politique, du point de vue de l'État comme de la société civile. Étendons les enseignements et les publications de la communauté académique à ces analyses en multipliant les postures réflexives sur la science économique étendue à son ingénierie. En second lieu ouvrons les formations, les séminaires de recherche, les colloques de la discipline économique à des approches d'autres disciplines et multiplions les points de rencontre avec les sociologues, les politologues, les historiens, les anthropologues, pour enrichir cette vision réflexive sur le rôle de l'économie dans la société. À ce titre, il est évident qu'il est plus important de poursuivre à l'université la réflexion globale engagée au lycée par les sciences économiques et sociales sur les rapports entre économie et société, que d'anticiper au lycée une formation théorique à la science économique standard. Il est tout aussi urgent que nos économistes soient formés non comme des spécialistes mais comme les futurs "ingénieurs sociaux " qu'ils seront, en privilégiant le pluralisme des approches théoriques et des méthodes, et en fuyant tout dogmatisme et enseignement magistral au profit d'une pédagogie de type problem oriented qui prend sa source dans la cité et y retourne. Enfin, il faut consacrer du temps et des espaces au troisième pilier de l'expertise, à savoir le débat citoyen : tout positionnement d'expertise qui procède d'un calcul savant articulé au seul calcul politique des décideurs qui ne fait pas l'objet d'un débat démocratique sera voué à l'échec par un biais technocratique qui laissera de côté des problématiques et des intérêts portés par les populations ciblées par les politiques. La seule force qui peut pousser des scientifiques à s'occuper de politique c'est de savoir que cette politique est celle de l'intérêt général, et pas seulement la résultante d'un jeu (équilibré ou déséquilibré) des intérêts privés. La crise idéologique du néolibéralisme qui a succédé à la crise économique et financière de 2007-2009 et les soulèvements populaires des pays arabes de l'hiver 2010-2011 devraient nous 
aider à retrouver le chemin démocratique d'une nécessité de construire ensemble les règles et les usages de nos sociétés. Retournons le constat autiste de 2000 : oui la science économique peut être euclidienne et parler du monde " réel »; oui elle est capable de formater et transformer profondément les cadres de notre vie sociale; oui elle peut nous aider à revendiquer une volonté politique, à orienter la trajectoire de nos rapports à l'environnement, c'est-à-dire aux autres et à ce qu'il reste du monde naturel.

\section{Bibliographie}

Aglietta, Michel et Antoine Rebérioux, Dérives du capitalisme financier, Paris, Albin Michel, 2004.

Armatte, Michel, Histoire du Modèle linéaire. Formes et usages en Statistique et en Économétrie jusqu'en 1945, Thèse de Doctorat, EHESS, sous la dir. de Jacques Mairesse, 1995.

Armatte, Michel, "Crise financière : modèles du risque et risque de modèle ", Mouvement, 2009, sur le site : www.mouvements.info/crise-financieremodeles-du-risque.htm, consulté en janvier 2011.

Armatte, Michel, La science économique comme ingénierie. Quantification et modélisation, Paris, Presses des Mines, 2010.

Armatte, Michel et Amy Dahan, «Modèles et modélisations (1950-2000) : nouvelles pratiques, nouveaux enjeux ", Revue d'Histoire des Sciences, vol. $57, \mathrm{n}^{\circ} 2$, 2004, p. 245-305.

Artus, Patrick et al., La crise des subprimes, Rapport du Conseil d'Analyse Économique, Paris, La Documentation Française, 2008.

Beck,Ulrich, La société du risque. Sur la voie d'une autre modernité, Paris, Aubier, 2001.

Bensaude-Vincent, Bernadette, Les vertiges de la techno-science. Façonner le monde atome par atome, Paris, La Découverte, 2009.

Bijker, Wiebe, Thomas P. Hughes et Trevor Pinch (dir.), The Social Construction of Technological Systems: New Directions in the Sociology and History of Technology, Cambridge (MA), M.I.T. Press, 1987.

Bloor, David, Sociologie de la logique ou les limites de l'épistémologie, 
trad. Ebnöther, Paris, Pandore, 1982 [1976].

Callon, Michel, "Éléments pour une sociologie de la traduction. La domestication des coquilles Saint-Jacques et des marins-pêcheurs dans la baie de Saint-Brieuc ", L’Année Sociologique, no 36, 1986, p. 169208.

Callon, Michel, The Laws of the Markets, Londres, Blackwell, 1998.

Callon, Michel, "Performative Economics ", dans Donald MacKenzie, Fabian Muniesa et Lucia Siu (dir.), Do Economists Make Markets, Princeton, Princeton University Press, 2007, p. 311-357.

Callon, Michel, "The Role of Hybrid Communities and Socio-Technical Arrangements in the Participatory Design ", Journal of the Center for Information Studies, vol. 5, no 3, 2004, p. 3-10.

Callon, Michel, Pierre Lascoumes et Yannick Barthes, Agir dans un monde incertain. Essai sur la démocratie technique, Paris, Seuil, 2001.

Dahan, Amy et Dominique Pestre (dir.), Les Sciences dans et pour la Guerre, EHESS, Paris, 2004.

Dahan-Dalmedico, Amy, (dir.), Les modèles du futur, Paris, La Découverte, 2007.

Desrosières, Alain, La politique des grands nombres. Histoire de la raison statistique, La Découverte, $2^{\mathrm{e}}$ éd., 2000 [1993].

Desrosières, Alain, Problèmes économiques, $\mathrm{n}^{\circ}$ 2725, 29 août 2001, p. 26-32.

Desrosières, Alain, «L'État, le marché et les statistiques : cinq façons d'agir sur l'économie ", Courrier des Statistiques, ${ }^{\text {os }}$ 95-96, décembre 2000, p. 3-10.

Desrosières, Alain, Pour une sociologie historique de la quantification, L'argument statistique 1, Presses de l'École des Mines, 2008.

Desrosières, Alain, Gouverner par les nombres, L'argument statistique 2, Presses de l'École des Mines, 2008.

Etner, François, Histoire du calcul économique en France, Paris, Economica, 1987.

Fama, Eugene, "The Behavior of Stock Market Prices ", Journal of Business, $\mathrm{n}^{\circ}$ 38, Janvier 1965, p. 34-105.

Fama, Eugene, "Efficient Capital Market: II ", Journal of Finance, 25 décembre 1970, p. 338-417.

Fitoussi, Jean-Paul, L'enseignement supérieur des sciences économiques en question, Rapport au Ministre de l'Éducation nationale, Paris, Fayard, 2001.

Gibbons, Michaël et al. (dir.), The New Production of Knowledge: The 
Dynamics of Science and Research in Contemporary Countries, London and Thousand Oaks, SAGE, 1994.

Hughes, Thomas P. et al. (dir.), The Social Construction of Technological Systems: New Directions in the Sociology and History of Technology, Cambridge (MA), M.I.T. Press, 1987.

Jorion, Paul, La crise. Des subprimes au séisme financier planétaire, Paris, Fayard, 2008.

Jurdant, Baudoin (dir.), Impostures scientifiques. Les malentendus de l'affaire Sokal, Paris, La Découverte, 1998.

Latour, Bruno (dir.), La science telle qu'elle se fait : anthologie de la sociologie des Sciences de langue anglaise, Paris, Pandore, 1982.

Latour, Bruno, Les microbes: guerre et paix, suivi de Irréductions, Paris, Métaillé, 1984.

Le Monde, "Entretien avec Jean-Pierre Jouyet ", 9/12/2010.

Lordon, Frédéric, Jusqu'à quand? Pour en finir avec les crises financières, Paris, Raison d'Agir, 2008.

MacKenzie, Donald, Fabian Muniesa et Lucia Siu, Do Economists Make Markets?, Princeton, Princeton University Press, 2007.

Masse, Pierre, "Application des probabilités en chaîne à l'hydrologie statistique et au jeu des réservoirs ", Journal de la Société de Statistique de Paris, 1944, p. 204-219.

Morgan, Mary S., The History of Econometrics Ideas, Cambridge, Cambridge University Press, 1989.

Morgan, Mary S. et Margaret Morrison, Models as Mediators. Perspectives on Natural and Social Science, Cambridge, Cambridge University Press, 1999.

Munagorri, Encinas de (dir.), Expertise et gouvernance du changement climatique, Paris, LGDJ, 2009.

Muniesa, Fabian, "Un robot walrasien. Cotation électronique et justesse de la découverte du prix ", Politix, vol. 13, n 52, 2000, p. 121-154.

Muniesa, Fabian, Des marchés comme algorithmes. Sociologie de la cotation électronique à la Bourse de Paris, Thèse de Doctorat, Paris, École des Mines, 2003.

Nordon, Didier, Les mathématiques pures n'existent pas, Paris, Actes Sud, 1993 [1981].

Orléan, André, De l'euphorie à la panique : penser la crise financière, Paris, Éditions de la rue d'Ulm, coll. «opuscule du cepremap » 2009.

Pestre, Dominique, Science, argent et politique. Un essai d'interprétation, Paris, INRA, 2003. 
Porter, Theodore M., Trust in Numbers. The Pursuit of Objectivity in Science and Public Life, Princeton, Princeton University Press, 1995.

Sapir, Jacques, "Une décade prodigieuse. La crise financière entre temps court et temps long ", Revue de la régulation, $2^{\mathrm{e}}$ semestre 2008, http:// regulation.revues.org/document 4032 .html, site consulté en décembre 2010.

Shapin, Steven, 1998, La révolution scientifique, Flammarion; traduit de The scientific revolution, Chicago, 1996.

Touffut, Jean-Philippe (dir.), Changement de climat, changement d'économie, Centre Cournot, Albin Michel, 2010.

Vatin, François, Le travail, sciences et société, Université de Bruxelles, 1999.

Vatin, François, Économie des forces et production d'utilité. La pensée gestionnaire des ingénieurs des ponts (1831-1891). Edition de la thèse de Bernard Grall (1962-1997), Rennes, Presses universitaires de Rennes, 2004.

Walliser, Bernard, "La science économique ", dans Jean-Michel Berthelot (dir.), Épistémologie des sciences sociales, Paris, PUF, 2001, p. 117147.

Walter, Christian et Eric Brian (dir.), Critique de la valeur fondamentale, Springer, 2007.

Walter, Christian et Michel de Pracontal, Le virus B. Crise financière et mathématiques, Paris, Le Seuil, 2009.

Weintraub, E. Roy, How Economics Became a Mathematical Science, Durham and London, Duke University Press, 2002. 